Sustainable Forestry (2019) Volume 2

doi:10.24294/sf.v1i3.955

\title{
Taiwan Unlawful Remediation Approach
}

\section{T. Y. Yeh" ${ }^{1 *}$, Min-Hao Wu ${ }^{1}, K_{\text {F Chen }}{ }^{2}$, Y P Peng ${ }^{3}$}

${ }^{1}$ Department of Civil and Environmental Engineering, National University of Kaohsiung, Taiwan, Tel: 886-7-591-9536, Fax: 886-7-591-9376, E-mail: tyyeh@nuk.edu.tw

${ }^{2}$ Department of civil engineering, National Chi Nan University

${ }^{3}$ Department of environmental engineering. Tunghai University

\begin{abstract}
Soil and groundwater remediation act has been enacted and executed since year 2000 in Taiwan. It has been ten good years till today where lots of remediation techniques progressively employed to improve Taiwan soil and groundwater resource quality. Regulatory agencies, academia, remediation consulting firms, on-site professional engineers all have contribute the proud ten years in terms of soil and groundwater clean-up contribution. However, some of technologies were un-environmental friendly even detrimental and damage to Taiwan precious soil and groundwater resources. In Article one of the current Taiwan soil and groundwater Act, it clearly stated that soil is a precious nature resources. Soil definitely is not a waste, shame on us most of current most commonly employed remediation are unlawful and merely aiming to save time and money consideration without any care to our land. Dig-and-dump and soil acid washing are damaged employed in almost every single local environment agency soil clean-up project. Lot of money, effort and time has been spent during past ten years. Most of the spending is not improving soil quality using Green approach.
\end{abstract}

Keywords: Soil and Groundwater Remediation Act; Dig-and-dump; Soil Acid Washing

\section{Introduction}

Soil and groundwater remediation act has been enacted and executed since year 2000 in Taiwan. It has been ten good years till today where lots of remediation techniques progressively employed to improve Taiwan soil and groundwater resource quality. Regulatory agencies, academia, remediation consulting firms, on-site professional engineers all have contribute the proud ten years in terms of soil and groundwater clean-up contribution. However, some of technologies were un-environmental friendly even detrimental and damage to Taiwan precious soil and groundwater resources. In Article one of the current Taiwan soil and groundwater Act, it clearly stated that soil is a precious nature resources. Soil definitely is not a waste, shame on us most of current most commonly employed remediation are unlawful and merely aiming to save time and money consideration without any care to our land. Dig-and-dump and soil acid washing are damaged employed in almost every single local environment agency soil clean-up project. Lot of money, effort and time has been spent during past ten years. Most of the spending is not improving soil quality using Green approach.

It is really confusing regarding the lesson learned and gained while used these chemical physical, totally un-environmental friendly treatment techniques. Two remediation approaches, namely dig-and-dump and soil acid washing simply treat soil as garbage, waste, and junk, not the soil law indicated that soil is a resource. The purpose of this paper is aimed to raise all you concerns and care toward our precious soil property, toward remediation engineers and particularly some of those outdated governmental authorities in my resident juridical area who have never taken so far a deep thought of current serious situation regarding soil damage.

A novel green remediation approach intends to convey in this paper by employing plant to gradually reduce soil metal contamination through several rounds of planting and harvesting. Unlike phytoextraction, phytoattenuation aimsto reduce soil metal pollution in a gradually and less aggressive approach such as chelator assisted remediation. The initial pollution level generally is lower than most soil contamination sites. Therefore, plant is easier to propagate to

Copyright (C) 2019 T. Y. Yeh et al.

doi: 10.24294/sf.v1i3.955

EnPress Publisher LLC.This work is licensed under the Creative Commons Attribution-NonCommercial 4.0 International License (CC BY-NC 4.0).

http://creativecommons.org/licenses/ by/4.0/ 
increase biomass inducing reliable metal uptake. The conceptual model is shown in Figure 1.
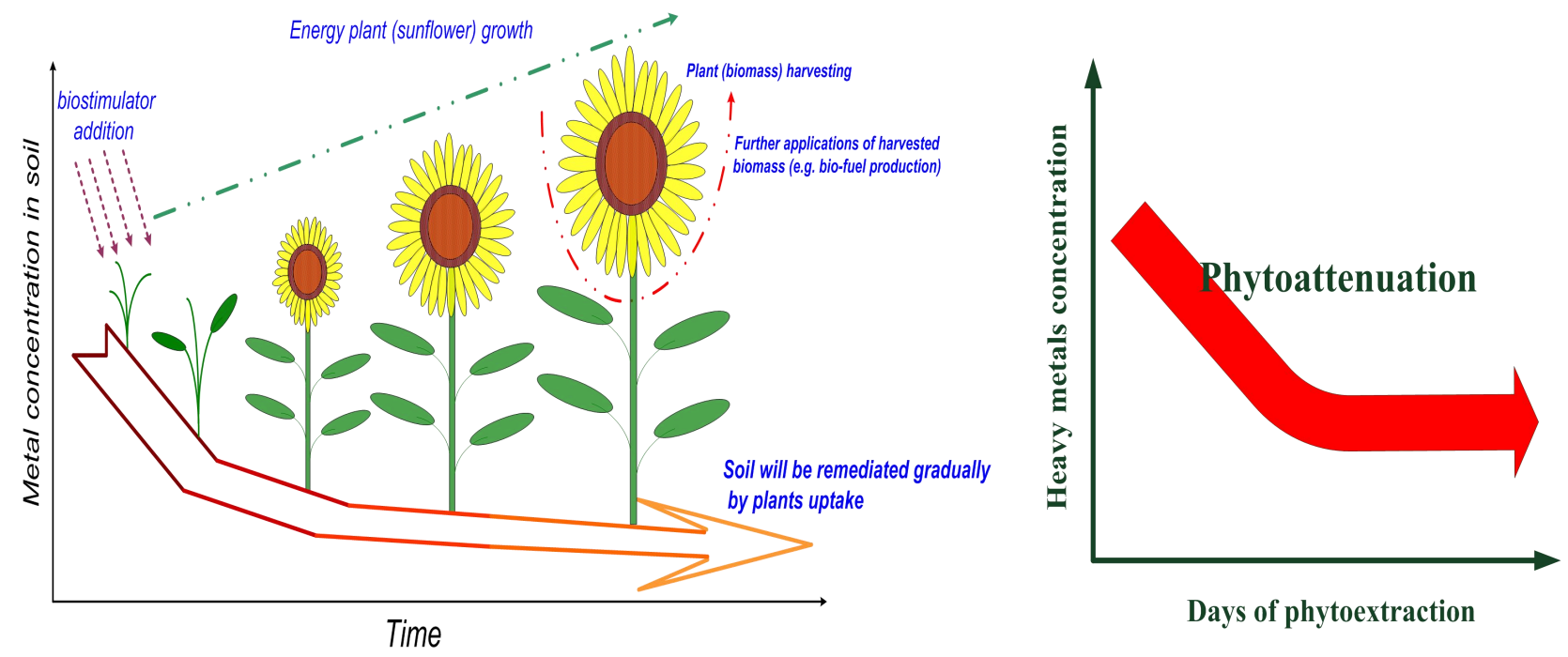

Figure 1; The schematic of pytoattenuation.

Attenuation is borrowing from the concept "natural attenuation" which has been commonly proposed as a remediation approach for organic pollutants such as DNAPL (dense non-aqueous liquid) solvent TCE (tri-chloro ethylene) and PCE (tetra-chloro ethylene) or LNAPL (light non-aqueous liquid) petroleum product BTEX (benzene, toluene, ethyl benzene, and xylene. Natural attenuation mainly used natural pollution mitigation mechanism including microbial degradation, adsorption, volatilization, etc. This approach is targeted to pollutant which is not degraded in a reasonable time using conventional remediation techniques, technical imperfectability, or the cost beyond the affordable monetary amounts, economical imperfectability.

$\mathrm{Cu}$ is used as the fodder additives for preventing swine diarrhea and skin abrasion (Yeh and $\mathrm{Wu}, 2009$ ). $\mathrm{Cu}$ has been reported the toxicity to phytoplankton and been employed as algaecide for serious eutrophication mitigation. The careless management of $\mathrm{Cu}$ wastewater from swine industries could damage the water and soil environment.

The choice of plant is more flexible than phytoextration. Plant is not necessary to be a hyperaccumulator and biomass production is not required to be enormous. Using several sessions of agricultural planting and harvesting, the metal contamination is gradually to reduce to an acceptable soil background concentration. The only concern is the time requirement for the whole attenuation operation. If the site has the emergent health and ecological damage concern, the aggressive remediation takes into the substation list to be conducted to ensure public health and ecological protection.

Possible ideal plants include wetland water pollution mitigation macrophytes such as vetiver, cattail, and reed which has been demonstrated to be easily propagation and capable to reduce water and sediment metal levels (Yeh et al., 2010). The harvested plant wastes should be properly managed to prevent the secondary environmental contamination. An alternative plant is the energy macrophytes such as sunflower and Chinese cabbage. After harvesting, the residue plant can be resued to produce bio-fuel which is green and substitute to petroleum fuels to lesson current energy concern.

Vetiver is known for its effectiveness in soil erosion control due to its unique morphological and physiological characteristics. Vetiver is also a high biomass plant with remarkable photosynthetic efficiency that renders it tolerant against various harsh environmental conditions. Vetiver with deep-rooted and higher water-use can effectively stabilize soluble metals in soils. These properties enable vetiver to be an ideal candidate for phytoattenuation and have been investigated in the study.

Sunflower (Helianthus annuus) is a fast-growing crop that has been commonly used for phytoextraction of metal contaminated soils. Sunflower has the potential as biofuel to become the substitute of fossil fuels, especially the increasing oil prize in recent years. The higher biomass production of sunflower, contribute them being the candidates 
of phytoextration contaminant and then harvested as potential fuel substitution.

Dig and dump generally wasted numerous of energy and manpower than green remediation approaches. The dig out soil might need an appropriate final disposal to dipose the highly comtaminated waste, however in Taiwan the waste was roudly treated and dump. A environmental friendly mitigation technique need to be employed while phtoextration will be a viable one ( et al.). Phytoattuation was not aimed to reduce high contaminated soil rather medium and low level pollution.

Plant speciese are crucil to achieve successful remediation. Wetland macrophte vetiver, cattail, reed and hyperaccumulate species wwer commonly used. Recently energy plant sunflower and Chiners cabbage are also employed. Prominant results have all been demonstation to prove green remediaton effectiveness.

The objective of this study is to study sunflower and Chinese cabbge effectiveness to remove $\mathrm{Cr}$ and $\mathrm{Pb}$ by enhanced by biopromoter IAA and GA3. SEM, EDX, and FTIR will be conducted to reveal the plant surface propreties while PCRDGGE will also be investigated to show the microbial community of root environment. The results of this study can be used for other investigation.

\section{Results and Discussion}

\subsection{Background soil concentration including total metal and metal fractionation}

The sequential extraction results can be used to depict metal mobility. Generally, the latter three fractions, namely organic, Fe- and Mn-hydroxide, and sulfide are more inert, permanently bound to soils, and less bioavailable while first two fractions including exchangeable and inorganic have been defined as loosely bound (Yeh et al, 2009).

\subsection{The biostimulator vetiver propagation enhancement and $\mathrm{Cu}$ soil reduction}

IAA and GA3 both performed satisfactory vetiver growth enhancement relative to control. GA3 generally possessed better propagation upgrade than IAA. Both biostimulator could employed for vetiver growth increase which is the merit for further plant uptake. Biostimulators commonly employed in agricultural amendment to enhance produce propragation. Appling in contaminated site mitigation was unpresedent and the results were optimistic. The price the addition biostimuator needs to further evaluated.

\subsection{Phytoattenuation evaluation}

The results of the attenuation study using vetiver and two stimulators have demonstrated prominent success. After 4 cycles of planting and harvesting $\mathrm{Cu}$ levels had demonstrated gradually metal decreasing. The control, stimulator, and stimulator remaining copper levers were progressingly decended. These results were very effective and indicted that phytoattenuation can be a green alternative to mitigate soil metal contamination with or without biostimulator assistant.

\subsection{Phytoattenuation evaluation}

Four stage of phytoattenuation observation demonstrated positive medium to low contaminated level soil mitigation which can be used for further similar site application. Phytoattenuation though is not effective for high level metal contamination while it is environmental friendly without using dig-and -dump rather EDTA chelation enhanceing expected to be well received worldwide.

\subsection{PCR analysis}

Technologies include: gene probes, microarrays, and conventional and real-time polymerase chain reaction (PCR), whole genome amplification, sequencing, comparative genomics, and metagenomics. Together these proved unprecedented ability to interrogate the microbial world.

Detection of a specific gene target has become routine practice i most molecular baboartaoies. The ability to analyze 16S rRNA gene sequence using PCR has rsulted in a virual eaploprerfomeed sample with prior DNA extract form environmental sample. PCR has revolutionized the ability of researches to detect specific nucleic nuclear acid sequences in isolated as well in environmental samples. PCR is extremely sanative able in some cased o detect a single target molecuse. PCR assay has been optimized, it is relative fast to get results in only a few hours, to several days or weeks that may be necessary for cultural methods. 


\section{Conclusion}

Vetiver has been demonstrated as valid plant for phytoattenuation ideal plant due to it is great biomass prorogation and metal prominent uptake. This study has demonstrated after several sessions of vetiver planting and harvesting. Biostimulators, $\mathrm{GA}_{3}$ and IAA, have demonstrated effective plant propagation enhancement. $\mathrm{Cu}$ descending levels were statistically significant relative to the control. The soil metal level reduction achieved acceptable levels. More rounds of planting and harvesting, the soil metal concentrations expected to be much lessoned in real sites. Green remediation concepts such as phytoattenuation and phytoextration need to be taken as serious concern.

\section{Reference}

1. Bunluesin, S., Kruatrachue, M., Pokethitiyook, P., Upatham, S., Lanza, G. R. (2007). Batch and Continuous packed column studies of cadmium biosorption by Hydrilla verticillata biomass. Journal of Bioscience and Bioengineering, 103(6), 509-513

2. Fritioff, A., Greger, M. (2006). Uptake and distribution of $\mathrm{Zn}, \mathrm{Cu}, \mathrm{Cd}$, and $\mathrm{Pb}$ in an aquatic plant Potamogeton natans. Chemosphere, 63, 220-227.

3. Grispen, V. M. J., Nelissen, H. J. M., Verkleij, J. A. C. (2006). Phytoextraction with Brassica napus L.: A tool for sustainable management of heavy metal contaminated soils. Environmental Pollution, 144, 77-83. 